In the PDT experiment on human RA synovial biopsies, the groups incubated with $28 \mathrm{H} 1-700 \mathrm{DX}$ and exposed to light showed apparent cell death in the synovial tissue as evidenced by the positive staining of both the $\mathrm{gH} 2 \mathrm{AX}$ and caspase 3 markers (figure $\mathrm{J}$ and $\mathrm{K}$ ). Staining of these markers co-localised with areas of high FAP staining (figure L). This was not the case in the control samples that were not exposed to either $28 \mathrm{H} 1-700 \mathrm{DX}$ and/or light (figure $\mathrm{A}-\mathrm{I}$ ). All biopsies did show FAP staining indicating that the cell death was only achieved when the biopsies were exposed to both the antibody and the light.

Conclusions: We have demonstrated fibroblast-specific cell death by targeted PDT using 700DX-conjugated $28 \mathrm{H} 1$. Furthermore, we demonstrated that PDT also induces cell death of FAP-positive cells in synovial tissue from RA patients, suggesting FAP-targeted PDT as a promising new tool in treating RA.

Disclosure of Interest: None declared

DOI: 10.1136/annrheumdis-2018-eular.6644

\section{SAT0062 METABOLOMICS AND METABOLIC FUNCTION ANALYSIS OF THE SECRETOME OF ARTICULAR CARTILAGE AND CHONDROCYTES IN RESPONSE TO PRO-INFLAMMATORY CYTOKINES}

C. Fellows $s^{1,2}$, H. Quasnichka ${ }^{1,2}$, N.R. Chowdhury ${ }^{3}$, E. Budd ${ }^{1,2}$, D.J. Skene ${ }^{3}$, A. Mobasheri $1^{1,2}$. ${ }^{1}$ Veterinary Pre-Clinical Studies; ${ }^{2}$ The D-BOARD European Consortium for Biomarker Discovery; ${ }^{3}$ Chronobiology, University of Surrey, Guildford, UK

Background: Chondrocytes rely primarily on glycolysis to meet their energy requirements, but can support cell survival and matrix synthesis during periods of nutrient stress by enhancing glycolysis with mitochondrial respiration. Accessing this 'spare respiratory capacity' requires optimal mitochondrial function. Impaired mitochondrial function is implicated in osteoarthritis $(O A)$. Metabolic adaptation is evident in early-stage $O A$, however cartilage from late-stage disease does not seem to have this flexibility. A deeper understanding of these complex metabolic pathways may identify new markers of disease stage, and support therapeutic strategies for treating $\mathrm{OA}$

Objectives: Metabolomics has the potential to reveal pathological pathways and identify novel biomarkers. The aim was to identify metabolic processes involved in early stage disease by analysis of metabolites and metabolic function in proinflammatory models of cartilage degradation.

Methods: Macroscopically normal articular cartilage was obtained from equine and bovine metacarpophalangeal joints. Equine cartilage explants $(n=6)$, and primary chondrocytes seeded at $105,000 / \mathrm{cm}^{2}(n=4)$, were cultured for 7 days in serum-free DMEM (Gibco) with or without $10 \mathrm{ng} / \mathrm{ml}$ equine interleukin-1 $\beta$ (IL-1 $\beta$ ) and $10 \mathrm{ng} / \mathrm{ml}$ tumour necrosis factor- $\alpha($ TNF- $\alpha)$. Secretome metabolite levels were measured using AbsoluteIDQ p180 targeted metabolomics kit (Biocrates), with Waters Xevo TQ-S mass spectrometer coupled to an Acquity UPLC system. PCA and OPLS-DA were performed using SIMCA-P v12.0 software. Metabolic function of primary equine $(n=9)$ and bovine chondrocytes $(n=3)$ was determined using Seahorse XFp and XFe24 analyzers. Cells were treated with species-specific $10 \mathrm{ng} / \mathrm{ml} \mathrm{IL-1} \beta$ and/or $10 \mathrm{ng} / \mathrm{ml}$ TNF- $\alpha$ for 18 hour, and metabolically challenged with the Mito Stress Test. Metabolite levels, and oxygen consumption rates, were normalised to total cell protein, and values analysed by ANOVA with Tukey's multiple comparison post-tests.

Results: Cytokine treatment decreased proline, ornithine and alpha-aminoadipic acid $(p<0.0001)$ in explant secretome. Citrulline increased with cytokine treatment $(p<0.0001)$ and glutamate, present in DMEM, was also elevated $(p<0.0001)$. Metabolomic analysis of chondrocyte secretome showed that glutamine decreased $(p<0.02)$ with cytokine treatment whereas citrulline was elevated $(p<0.003)$. Metabolic analysis showed that cytokine treatment reduced basal respiration and negated spare respiratory capacity in chondrocytes $(p<0.01)$, and the effect was due to IL-1 $\beta$ alone.

Conclusions: Explant metabolites which decreased with cytokine treatment are all downstream of glutamate. With elevated glutamate, this suggests that cytokines inhibit glutamate uptake and metabolism. Elevated citrulline in cell and explant models may be attributed to disruption of the urea cycle via induction of nitric oxide synthase. IL-1 $\beta$ alone negated spare respiratory capacity, and chondrocytes remained glycolytic. In conclusion, cytokines disrupt glutamate and citrulline metabolism, normally tightly regulated mitochondrial pathways, and IL-1 $\beta$ alone is responsible for the metabolic switch. These metabolic pathways could provide markers of early-stage inflammatory disease.

Disclosure of Interest: None declared

DOI: 10.1136/annrheumdis-2018-eular.6426

\section{SAT0063 \\ EFFECTS OF INTRA-ARTICULAR INJECTED DIACEREIN- LOADED NANOPARTICLES ON JOINT IN RAT MODEL OF OSTEOARTHRITIS}

J.H. Jung, G.G. Song, S.J. Choi, J.D. Ji, Y.H. Lee, J.-H. Kim. Internal Medicine, Korea University Medical Center, Seoul, Korea, Republic Of

Background: Osteoarthritis $(O A)$ is a major health problem in recent years, but the current medical treatment is mainly symptom control and joint disability improvement. Diacerein (DIA) reduces the level of interleukin (IL) - 1 receptor on the surface of chondrocytes, alleviates the pain, and prevents the structural deg radation of the joint tissue. However, DIA has the side effects such as diarrhoea and urine discoloration when taken orally. To increase bioavailability and reduce systemic side effects, it is preferable to inject DIA directly into the joints. Nanoparticulate drug carriers have been attempted with the advances in drug delivery systems.

Objectives: We investigated that DIA-loaded nanoparticles (DIA/NPs) can efficiently inhibit the inflammatory reaction in synoviocytes stimulated by lipopolysaccharide in vitro and alleviate both inflammation and cartilage degeneration in monosodium iodoacetate (MIA)-induced OA rat model in vivo.

Methods: DIA/NPs were fabricated by water/oil/water emulsion method. In vitro, the mRNA levels of pro-inflammatory cytokines (IL-1, IL-6, MMP-3, MMP-13, COX-2, ADAMTS-5, and TNF- $\alpha$ ) were measured at 1 st and $3 r d$ day after the administration of NPs only and DIA/NPs to synoviocytes using real-time PCR. MIA was intra-articular injected through the infrapatellar ligament of the rats' knee to induce $O A$. The rats were randomly divided into the six treatment groups: ${ }^{1}$ control, ${ }^{2} \mathrm{MIA},{ }^{3} \mathrm{MIA}$ and NPs, ${ }^{4} \mathrm{MIA}$ and DIA $(1 \%) / \mathrm{NPs},{ }^{5} \mathrm{MIA}$ and DIA(5\%)/NPs, and ${ }^{6}$ MIA and DIA(5\%) solution injection. After NPs, DIA(1\%)/NPs, DIA $(5 \%) / N P s$, and $\mathrm{DIA}(5 \%)$ solution were injected, at 8 th week, the rats were sacrificed to evaluate the plain radiographic and micro-computed tomography (micro-CT), histological study, and pro-inflammatory cytokines expression.

Results: The mRNA expression levels for pro-inflammatory cytokines in cells seeded with $\mathrm{DIA}(5 \%) / N P s$ and $\mathrm{DIA}(5 \%)$ solution were significantly lower than those in cells seeded with NPs at 1 st and 3rd day. Moreover, the mRNA levels of pro-inflammatory cytokines in cells injected with DIA(5\%) solution decreased much greater than in cells injected with $\mathrm{DIA}(5 \%) / \mathrm{NPs}$. The rats injected $\mathrm{DIA}(5 \%) /$ NPs showed the least amount of cartilage and bone damage on plain radiography and micro-CT and had the less cartilage loss and the bony erosions on microscopic observation. The pro-inflammatory cytokines expression was lowest in the rats injected $\mathrm{DIA}(5 \%) / \mathrm{NPs}$, followed by NPs, $\mathrm{DIA}(1 \%) / \mathrm{NPs}$, and $\mathrm{DIA}(5 \%)$ solution.

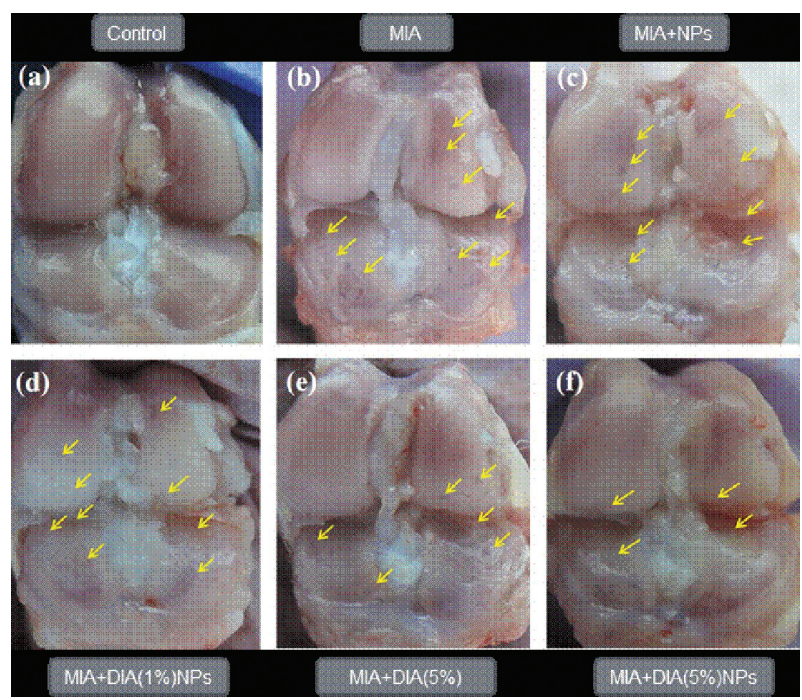

Abstract SAT0063 - Figure 1. The macroscopic observations for 9 weeks after the MIA injection and for 8 weeks after treated with NPs, DIA $(1 \%) / \mathrm{NPs}$, DIA $(5 \%) / \mathrm{NPs}$, or DIA $(5 \%)$ solution injections (arrows indicate erosions).

Conclusions: $\mathrm{DIA}(5 \%) / \mathrm{NPs}$ are a promising therapeutic material to control the symptoms and prevent the progression of $\mathrm{OA}$. 


\section{REFERENCES:}

[1] Jain A, et al. Diacerein protects against iodoacetate-induced osteoarthritis in the femorotibial joints of rats. J Biomed Res 2015;29:405-413.

[2] Bairwa K, et al. Nanoparticle formulation of 11-keto- $\beta$-boswellic acid (KBA): anti-inflammatory activity and in vivo pharmacokinetics. Pharm Biol 2016;54:2909-2916.

[3] Park JW, et al. Ibuprofen-loaded porous microspheres suppressed the progression of monosodium iodoacetate-induced osteoarthritis in a rat model. Colloids Surf B Biointerfaces 2016;147:265-273.

Acknowledgements: No grants or other support were received for the conduction of this study.

Disclosure of Interest: None declared

DOI: 10.1136/annrheumdis-2018-eular.1805

\section{SAT0064 CD14+CD16+ MONOCYTE SUBPOPULATION IS DOMINANT IN THE INFLAMMATION OF OSTEOARTHRITIS SYNOVIAL FLUID}

J. Kim ${ }^{1}$, S.-J. Yoo ${ }^{1}$, Y. Kim², S.W. Kang ${ }^{1}$, S.C. Shim ${ }^{1}$, S.M. Kim ${ }^{3}$. ${ }^{1}$ Internal Medicine, Chungnam National University College of Medicine; ${ }^{2}$ Internal Medicine, Daejon Veterans Hospital, Daejeon; ${ }^{3}$ Internal Medicine, Chungbuk National University Hospital, Cheong-ju, Korea, Republic Of

Background: In osteoarthritis (OA), the activation of inflammation response involving the interaction of cartilage and synovial hyperplasia may contribute to disease progression. However, inflammatory cells in OA synovial fluid (OASF) have been rarely studied.

Objectives: To investigate the phenotype of CD14 +cells and the secretion of proinflammatory cytokines by these cells

Methods: Immunohistochemistry staining in OA synovium was performed using anti-CD14, anti-CD16 and anti-CD56 antibody. The OASF was obtained through arthrocentesis. Mononuclear cells from OASFs were stained with anti-CD3, antiCD4, anti-CD14, anti-CD16, anti-TLR4 or anti-TLR2 and analysed using flow cytometry. CD14 +CD16+and CD14+CD16 mononuclar cells in OASF were selected with magnetic microbeads. In the supernatant of these cells culture, the concentration of IL-1 $\beta$, IL-6, IL-8, TNF $\alpha$, MMP-1, -3 were measured by Luminex.

Results: In OA synovium, CD14 or CD16 was stained, but CD56 was not expressed. In OASF, there was a substantial number of CD14+cells $(36.6 \%$ $\pm 25.2 \%)$, CD3 +cells $(37.4 \% \pm 12.9 \%)$, with a rarity of CD90 +cells $(1.7 \% \pm 1.3 \%)$. The proportion of CD14 +cells was increased significantly in recurred synovial fluid, compared with the proportion in initial synovial fluid. Among CD14 +cells in OASF, CD14 +CD16+monocyte subpopulation $(21.2 \% \pm 21.8 \%)$ was more abundant than CD14 +CD16 - monocyte subpopulation (10.9\% $\pm 10.0 \%)$. TLR4 and TLR2 expressions were higher in CD14 +CD16+cells than in CD14 +CD16 cells. The concentration of IL- 8 and MMP-3 was more increased in the supernatant of CD14 +CD16+cells than in that of CD14 +CD16 - cells.

Conclusions: In OASF, the proportion of CD14 +cells was increased in recurred synovial effusion. Compared to CD14 +CD16- monocyte subpopulation, CD14 +CD16+monocyte subpopulation released more cytokine such as IL-8 and MMP-3, and had higher expressions of TLR4 and TLR2.

Disclosure of Interest: None declared

DOI: 10.1136/annrheumdis-2018-eular.5140

\section{SAT0065 CHONDROCYTES UNDER PATHOLOGICAL CONDITIONS PREFERENTIALLY DEGRADES SELECTED TRNAS TO INHIBIT PROTEIN SYNTHESIS}

J. Green, T. Haqqi. North East Ohio Medical University, Rootstown, USA

Background: Oxidative and ER stress play an important role in the pathogenesis of osteoarthritis (OA). These stress conditions can induce the cleavage of transfer RNA (tRNA) via angiogenin. This cleavage at the anticodon loop of the tRNA produces 5'-tRNA and 3'-tRNA halves known as tiRNAs.

Objectives: We aim to identify specific tRNA to tiRNA isotype fragments that occur during oxidative or ER stress in human OA chondrocytes.

Methods: Primary human articular chondrocytes were enzymatically isolated from OA cartilage. Cells were cultured in vitro for 72 hour and then treated with tert-butyl hydroperoxide (TBHP) over a 12 hour time course or with Interleukin-1b (IL1b) over a 24 hour time course. RNA was collected for mRNA expression analysis (qRT-PCR). Protein was harvested for western blot analysis. A tRF and tiRNA PCR Array that profiles 55 known tiRNAs was used to perform an unbiased dissection of the formation of tiRNAs in OA chondrocytes. Total RNA was adapted using a First-Strand cDNA Synthesis Kit designed to create cDNA libraries from small RNAs for qPCR detection to identify ten novel tiRNAs associated with cell stress in OA chondrocytes.
Results: Using qRT-PCR RNase gene expression was measured. Of these RNases $250 \mu \mathrm{M}$ TBHP treatment at 12 hour induced KIAA0430 $(p<0.05)$, RNase7 $(p<0.0001)$ and ZC3H12A $(p<0.0001)$ but not angiogenin or SND1. $10 \mathrm{ng} / \mathrm{ml} \mathrm{IL1ß} \mathrm{treatment} \mathrm{at} 6$ hour induced SND1 $(p<0.0001)$, RNase $7(p<0.01)$, ZC3H12A $(p<0.01)$ but not angiogenin or KIAA0430. Chondrocytes undergoing oxidative or ER stress produced tiRNA halves in a time dependent manner, tiRNA formation was highest at 6 hour of IL1B treatment and after 12 hour of TBHP treatment. tiRNA fragments of tRNAs specific for arginine, glutamic acid, glycine, histidine, lysine and valine were increased after TBHP or IL1ß treatment. Ten tiRNAs that were induced by IL1B and TBHP were selected for further study. Primer sets for these tiRNA and the parent tRNAs were designed and screened across a wider time course, and with higher patient numbers.

Conclusions: The expression of several RNases was increased in a time dependent manner in stressed OA chondrocytes and may be involved in the cleaveage of tiRNA fragments. tiRNA halves were grouped together based on their parent tRNA amino acid target. We hypothesised that an increase in fold change in these grouped tiRNAs may result in less production of proteins that contain a high percentage of that specific amino acid. These changes in amino acid distribution may account for decreases seen in amino acid rich proteins such as Type I collagen (a glycine rich protein) in stressed chondrocytes. By designing tiRNA and parent tRNA primers we will discover if tiRNA formation effects total levels of parent tRNAs. The outcomes from this research will provide us with an increased understanding of tRNA to tiRNA formation in human chondrocytes and may allow us to identify therapeutic targets for the treatment of $\mathrm{OA}$ and provide insights into novel stress signalling pathways.

Acknowledgements: This work was supported by funds from the Northeast Ohio Medical University to $\mathrm{TMH}$.

Disclosure of Interest: None declared

DOI: 10.1136/annrheumdis-2018-eular.4874

\section{SAT0066 1 THE LONG NONCODING RNA (LNCRNA) HOTTIP IS A MASTER REGULATOR OF CELL CYCLE IN HAND SYNOVIAL FIBROBLASTS IN ARTHRITIS}

M. Frank Bertonceli ${ }^{1}$, T. Masterson ${ }^{1}$, E. Karouzakis ${ }^{1}$, C. Kolling ${ }^{2}$, A. Filer ${ }^{3}$, C. D. Buckley ${ }^{3}$, S. Gay ${ }^{1}$, O. Distler ${ }^{1}$, C. Ospelt ${ }^{1} .{ }^{1}$ Center of Experimental Rheumatology Zurich, University Hospital Zurich, Schlieren; ${ }^{2}$ Schulthess Clinik, Zurich, Switzerland; ${ }^{3}$ Institute of Inflammation and Ageing (IIA), University of Birmingham, Queen Elizabeth Hospital, Birmingham, UK

Background: Rheumatoid arthritis (RA) and other types of inflammatory arthritis follow a characteristic anatomical pattern of joint involvement. We have recently shown that local synovial stromal cells, specifically synovial fibroblasts, exhibit joint-specific transcriptomes and functions. In particular, hand SF exhibited prominent proliferative and chemotactic activities. Density of stroma and leukocyte infiltration were increased in hand synovium.

Objectives: To explore the role of hand/feet-specific IncRNA HOTTIP in shaping the function of hand synovial fibroblasts in arthritis.

Methods: We studied transcriptomes and epigenomes of hand, shoulder and knee SF from patients with RA or osteoarthritis and from knees of non-arthritic subjects using RNA-sequencing, Illumina HiSeq $2000 \mathrm{n}=21$ ), histone ChIPsequencing (Illumina HiSeq 2500, $n=7$ ) and Infinium HumanMethylation450 BeadChip $(n=12)$. qPCR was used to confirm RNA-sequencing data in a larger cohort of SF from different joints. We silenced the IncRNA HOTTIP in hand SF using LNA GapmeRs, followed by RNA-sequencing, qPCR, protein-protein inter action analysis of RNA-sequencing data (STRING), and in vitro assays for proliferation (BrdU assay) and apoptosis (Annexin V/PI staining).

Results: Genome-wide DNA methylation patterns and histone marks at actively transcribed DNA regions (H3K27ac) and enhancers (H3K4me1) defined jointspecific origin of SF. SF from hands and feet specifically expressed the IncRNA HOTTIP. This distal-specific HOTTIP expression coincided with the enrichment of H3K4me3 and H3K27ac and a decrease in repressive marks (H3K27me3, DNA methylation) at the HOTTIP promoter in hand SF. In contrast, the HOTTIP promoter displayed scarce activating, but abundant repressive epigenetic marks in shoulder and knee SF. Silencing of HOTTIP in hand SF altered the expression of 447 protein-coding genes ( $\log$ ratio $>|2|, F D R<0.05$ ). These genes were strongly enriched in the mitotic cell cycle protein interaction network $(n=48$ genes $p=3.3 \times 10-{ }^{7}$ ). Several of the enriched mitotic cell cycle genes, including NCAPG CENPO, ZWILCH and BUB1 were confirmed as downregulated by HOTTIP silencing in a larger cohort of hand SF $(n=6)$. The basal expression of 36 out of the 48 enriched cell cycle genes correlated with the basal HOTTIP expression in hand SF ( $n=6$, RNA-sequencing, $R>|0.06|)$. We further measured these correlations in a larger cohort of hand SF $(n=21)$ for a subset of the 36 genes using $9 P C R$ Among the measured genes, TADA 3 and CDC27 were confirmed to correlate with HOTTIP expression in hand SF $(R=0.5, p<0.05)$. Silencing of HOTTIP for 24 hour, 48 hour and 72 hour decreased the incorporation of BrdU into DNA of 